\title{
Validation of a new data-analysis software for multiple-peak analysis of $\gamma$ spectra at ISIS pulsed Neutron and Muon Source
}

\author{
L. Arcidiacono ${ }^{\mathrm{a}, \mathrm{b}, \mathrm{c}}$, A. Parmentier ${ }^{\mathrm{d}}, \mathrm{G}$. Festa ${ }^{\mathrm{c}, *}$, M. Martinón-Torres ${ }^{\mathrm{e}}$, C. Andreani ${ }^{\mathrm{a}}$, R. Senesi ${ }^{\mathrm{a}, \mathrm{f}}$ \\ a Universita' degli studi di Roma Tor Vergata, Physics department and Centro NAST via della Ricerca Scientifica 1, 00133 Rome, Italy \\ ${ }^{\mathrm{b}}$ University College of London, Institute of Archaeology 31-34 Gordon street, WC1H OPY London, United Kingdom \\ ${ }^{\mathrm{c}}$ Centro Fermi, Museo Storico della Fisica e Centro Studi e Ricerche 'Enrico Fermi', piazza del Viminale 1, 00184 Rome, Italy \\ d INFN-Division of Roma Tor Vergata, via della Ricerca Scientifica 1, 00133 Rome, Italy \\ ${ }^{\mathrm{e}}$ Archaeology department, University of Cambridge, Downy Street, CB2 3DZ Cambridge, United Kingdom \\ ${ }^{\mathrm{f}} \mathrm{CNR}-\mathrm{IPCF}$, sezione di Messina, Messina, Italy
}

\section{A R T I C L E I N F O}

\section{Keywords:}

ROOT software

PGAA

NRCA

Quantitative analysis

Epithermal neutrons

HPGe

\begin{abstract}
A B S T R A C T
In this work, we present a multi-peak fitting code in order to establish a new protocol for the analysis of $\gamma$ spectra at ISIS pulsed Neutron and Muon Source. The protocol, relying on the ROOT framework developed at CERN, has been tailored for the analysis of two specific gamma emitters $-{ }^{241} \mathrm{Am}$ and ${ }^{133} \mathrm{Ba}-$ and its results have been compared to those generated by Hypermet PC, a dedicated software package specifically devoted to the analysis of neutron induced $\gamma$-ray spectra and of widespread use since late 1990s. This new fitting procedure is scheduled for integration into the software for time-resolved prompt-gamma activation analysis, T-PGAA, currently under development at ISIS. T-PGAA allows for the simultaneous acquisition of photon energy and neutron time of flight, respectively, when prompt gamma rays are emitted by a sample after neutron absorption, essentially combining neutron resonance capture analysis (NRCA) and prompt gamma activation analysis (PGAA).
\end{abstract}

\section{Introduction}

In recent years, the use of neutron techniques at spallation neutron sources has been rapidly expanding with applications in the field of gamma spectroscopy that span from the characterisation of gamma background at neutron beamlines [1] to the isotopic analysis of materials [2].

In this context, one of the techniques commonly used for material characterisation exploits the pulsed nature of the source, specifically hinging upon the recovery of the time of flight of the absorbed neutron and emitted photon energy [3].

Prompt gamma activation analysis (PGAA) is a standard technique developed in the early 70s in Germany [4] and USA [5], and in more recent years it has been further improved at the Hungarian Institute of Isotopes in Budapest for the isotopic and elemental analysis of materials in a quantitative way [6].

We have recently developed a new simultaneous acquisition technique of the gamma energy spectrum and neutron time of flight in case of neutron irradiation of a sample: time-resolved prompt gamma activation analysis (T-PGAA) at ISIS pulsed Neutron and Muon Source [3]. This technique allows to enhance the isotopic sensitivity using epither- mal resonances for a selective quantitative analysis of intermediate- and heavy-mass isotopes.

In this framework, we are presenting here a code for multi-peak analysis tested on calibrated gamma sources based on the ROOT framework [7]. The results have been systematically compared to those obtained with Hypermet software, which has been considered, since the late 1990s, as the standard for an automatic and efficient analysis of multichannel pulse-height spectra at reactors [8,9]. Gamma spectra from the calibrated sources were recorded by a high purity germanium detector (HPGe), and processed using both the developed ROOT code and Hypermet software. The former was implemented following the peak-shape guidelines reported in [9], and resulting fitting parameters were compared to the homologous ones from the latter, finally attaining a good match that was interpreted as a first validation of the presented algorithm. This is intended as the starting point for the development of an integrated data analysis software tailored for T-PGAA technique at ISIS pulsed Neutron and Muon Source. This code has been developed with the aid of ROOT [7] in order to exploit the capability of this framework to handle files as large as 1TB, with a focus on flexibility and future software development.

\footnotetext{
* Corresponding author.

E-mail address: giulia.festa@centrofermi.it (G. Festa).
} 
Table 1

HPGe specifications.

\begin{tabular}{ll}
\hline & Detector details \\
\hline Material & HPGe, n-type \\
Crystal configuration & Coaxial \\
Crystal diameter & $59.7 \mathrm{~mm}$ \\
Cup length & $105 \mathrm{~mm}$ \\
Window thickness & $0.51 \mathrm{~mm} \mathrm{Be}$ \\
Crystal length & $79 \mathrm{~mm}$ \\
High Voltage bias & $-3800 \mathrm{~V}$ \\
Relative efficiency & $44.5 \%$ \\
Endcap diameter & $70 \mathrm{~mm}$ \\
Manufacturer and year & ORTEC 2013 \\
\hline
\end{tabular}
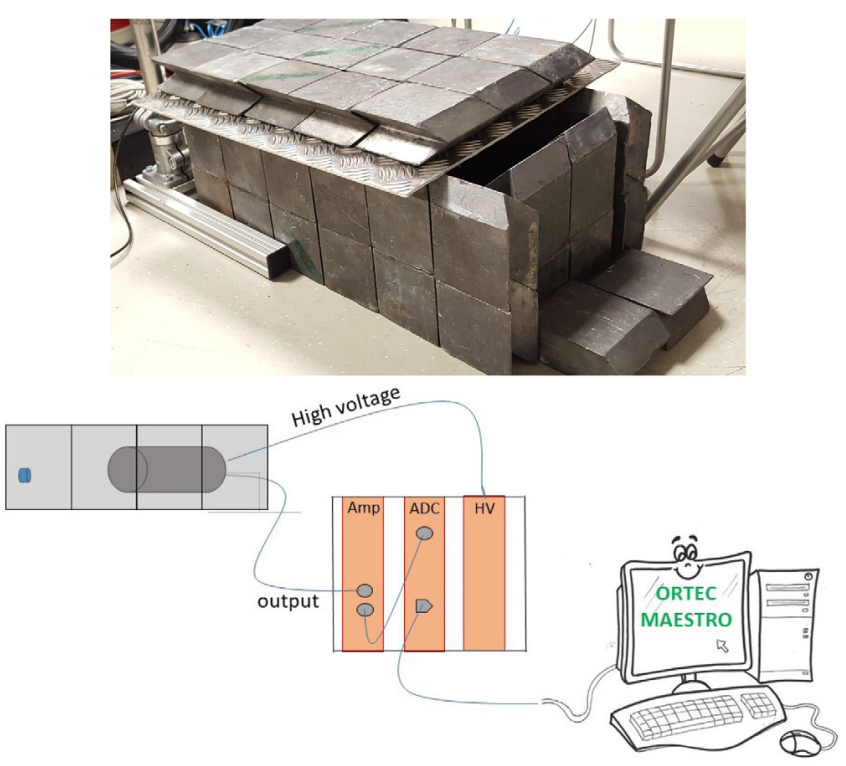

Fig. 1. Experimental setup. Top: lead shielding; bottom: schematic representation of the setup used for the measurements.

\section{Materials and methods}

The HPGe (see Table 1 for technical specifications) was cooled down to $77 \mathrm{~K}$ by means of an electric Ortec XCooler unit (https://www.orteconline.com), and it was used for the acquisition of a set of $\gamma$ spectra from two calibrated radionuclides in the radioprotection laboratory at ISIS pulsed Neutron and Muon Source, a low-background laboratory placed in Target Station 2 (TS2).

The HPGe was shielded by means of a cage of $10 \times 10 \times 5 \mathrm{~cm}^{3}$ pure-lead bricks in order to minimise the background (Fig. 1, top).

The setup (Fig. 1, bottom) is composed by:

- A high purity germanium detector GMX40P4-76RB;

- An Ortec $6595 \mathrm{kV}$ bias supply;

- An Ortec 572 Amplifier to amplify and reshape the signal into a quasi-Gaussian, and to select the energy ranges of interest;

- An Ortec 928 ADC to register the pulse-height signal in 16382 channels;

- A computer connected to the ADC and running Ortec MAESTRO software as user interface.

The description of major source features and acquisition parameters is reported in Table 2 .

Following recommendations reported in the 1996 IEEE protocol [10], we used discoidal standards presenting an active nucleus diameter $<2 \mathrm{~mm}$, and the acquisition time of both background and sources spectra was fixed. The standard distance between the detector and the source for this kind of measurements is usually $25 \mathrm{~cm}$, but in this
Table 2

Radioactive-source specifications. $\mathrm{A}_{0}$ is the reference activity reported in datasheets, $t_{1 / 2}$ is the half-life reported in LNE-LNHB/CEA nuclear data tables, $t_{\text {live }}$ is the livetime recorded by MAESTRO acquisition software; $\mathrm{d}$ is the source-detector distance.

\begin{tabular}{lllll}
\hline$\gamma$ sources & $\mathrm{A}_{0}[\mathrm{kBq}]$ & $\mathrm{t}_{\mathrm{l} / 2}[\mathrm{y}]$ & $\mathrm{t}_{\text {live }}[\mathrm{s}]$ & $\mathrm{d}[\mathrm{cm}]$ \\
\hline${ }^{241} \mathrm{Am}$ & $39.60 \pm 1.19$ & $432.6 \pm 0.6$ & 300 & $11.5 \pm 0.1$ \\
${ }^{133} \mathrm{Ba}$ & $36.60 \pm 1.10$ & $10.500 \pm 0.006$ & 300 & $11.5 \pm 0.1$ \\
\hline
\end{tabular}

case we shortened it to $11.5 \mathrm{~cm}$ - without considering the $2.6 \mathrm{~cm}$ between the endcap and the crystal - because of the low activities of the sources. The chosen distances constitute a good trade-off for the maximisation of the signal-to-noise $(\mathrm{S} / \mathrm{N})$ ratio and the minimisation of the coincidence summing, considering that no collimation or Comptonsuppression arrangements were implemented [11]. Such data were used for a thorough comparison between two analytical techniques in order to introduce and validate a new protocol for data analysis to be applied to T-PGAA data at spallation sources. The new software for data analysis was coded using ROOT, a C++-based suite extensively used in nuclear physics [7].

A raw data file and the associated background are fed as input to the code, then it performs a background subtraction and a further baseline correction to remove the residual environmental signal. At this point, the code performs the energy calibration with non-linearity correction included Fazekas et al. [12], and an automatic peak search in the IAEA database for isotopic labelling.

Peak search was implemented by means of the basic 1D function described in details in $[13,14]$. This function allows an automatic identification of spectral peaks in the presence of statistical fluctuations, starting from a user-defined threshold. The algorithm is based on smoothed second differences compared to their standard deviations. The IAEA database is read by the programme as an input pdf file, each peak centroid is compared to the energy list in the database, and a list of isotope candidates, with related cross sections, is returned as final output.

At this point, provided that a point-source geometry has been implemented and an energy range $[0: 2] \mathrm{MeV}$ has been spanned as in the present case, the code applies efficiency correction to current data using the $\epsilon_{i n t}$ function reported in [11]. Complete single-peak analysis follows.

In order to find the best fitting formula for the $\gamma$ peaks, we implemented the one used by Hypermet PC. The fitting formula we came up with for any peak profile in the $j$ th channel is:

$p(j)=\Gamma_{i} e^{-\left(\frac{j-j_{i, 0}}{\delta}\right)^{2}}+\frac{1}{2} A_{i} e^{\frac{j-j_{i, 0}}{\beta}} \operatorname{erfc}\left(\frac{\delta}{2 \beta}+\frac{j-j_{i, 0}}{\delta}\right)$

where the first addend on the left is a Gaussian of amplitude $\Gamma_{i}$ and variance $\frac{\delta}{\sqrt{2}}$, while the second addend is an exponentially modified Gaussian, of amplitude $A_{i}$, variance $\frac{\delta}{\sqrt{2}}$ and decay time constant $\beta$, which represents the peak left skewing (Fig. 2) regularly occurring towards lower energies because of incomplete charge collection in the detector crystal [11], an additional polynomial baseline correction is performed if required.

The fitting procedure embedded in our ROOT code is based on a simple fit that does not involve a matrix inversion, which could cause appreciable convergence difficulties in case of a large number of fitted parameters. Precisely, we chose to fit each peak individually rather than all together knowing that poor fitting can occur in case of peak overlapping. In $1 \mathrm{D}$ spectra, the quantity to be minimised in the fitting procedure is defined as follows:

$X^{2}=\frac{1}{N-M} \sum_{j=1}^{N} \frac{\left[y_{j}-f(j, a)\right]^{2}}{y_{j}}$

where $j$ is the channel in the fitted spectrum, $N$ is the number of channels, $M$ is the number of free parameters, $y_{j}$ is the experimental 


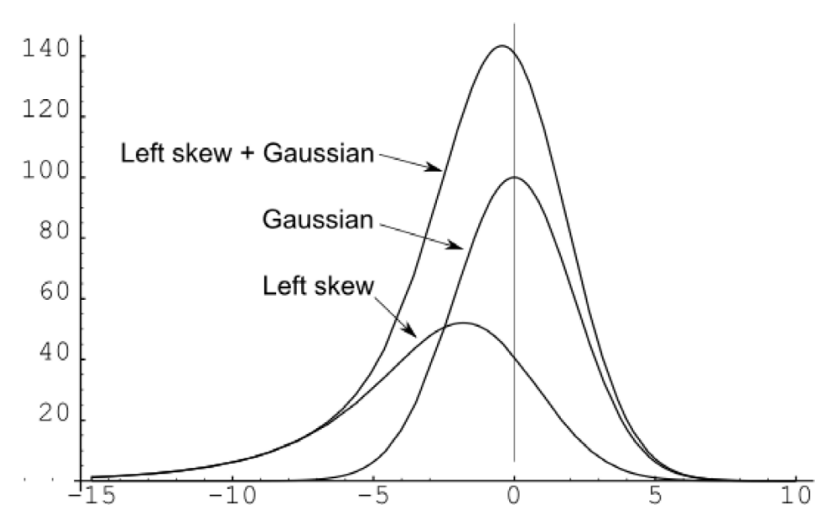

Fig. 2. Schematic representation of the left skew fitting in a gamma spectrum. Credits: http://hlabsoft.com/ HyperLabs software.

Table 3

Results obtained by ROOT-Hypermet comparison for 241Am.

\begin{tabular}{lllll}
\hline & Peak n. & Energy $[\mathrm{keV}]$ & FWHM $[\mathrm{keV}]$ & Peak area \\
\hline \multirow{2}{*}{ ROOT } & 1 & $25.84 \pm 0.05$ & $0.92 \pm 0.12$ & $2872.5 \pm 91.9$ \\
& 2 & $59.65 \pm 0.01$ & $0.97 \pm 0.01$ & $48766.4 \pm 633.9$ \\
\hline \multirow{2}{*}{ Hypermet PC } & 1 & $26.34 \pm 0.11$ & $0.92 \pm 0.04$ & $2681.0 \pm 83.1$ \\
& 2 & $59.65 \pm 0.01$ & $0.98 \pm 0.02$ & $47372.0 \pm 615.8$ \\
\hline
\end{tabular}

value in the $j$ th channel, $f(j, a)$ is the fitting formula. A detailed description of the fitting procedure reproduced through our ROOT code can be recovered in [15].

Within peak analysis, we calculated the Full Width Half Maximum (FWHM) and the integral of each peak. These are the quantities we compared to those obtained through Hypermet PC in order to demonstrate the applicability of the new ROOT software for data analysis, which was applied to simple gamma spectra in this case, but whose ultimate aim is full implementation in qualitative and quantitative analysis of Time-resolved Prompt Gamma Activation Analysis spectra.

\section{Results and discussion}

In order to assess the validity of the new data analysis software, we made a comparison between Areas, FWHM and calibrated energies obtained applying the standard Hypermet PC software and ROOT code to each spectral peak of two calibrated gamma-ray sources, whose reference activities are reported in Table 2. For the sake of consistency, the same normalisations were implemented with both methods. In the case of ${ }^{241} \mathrm{Am}$, ROOT-Hypermet comparison is reported in Table 3. As to ${ }^{133} \mathrm{Ba}$ (which is a nuclide whose spectrum comprises 9 emission lines), analogous results appear in Table 4.

We can notice that most peaks calculated via ROOT are smaller than their Hypermet-based counterparts; this may descend from effects of the bin-per-bin background subtraction possibly coupling to the use of only one multi-emitter $\left({ }^{133} \mathrm{Ba}\right)$ in the course of the efficiency calibration procedure, which may impact on non-linearity correction especially at high energies [11]. For the sake of comparison, we performed a quantitative test starting from spectral fits performed via both ROOT and Hypermet and retrieving the following quantities

1. The activity $A_{\text {meas }}(t)$ during measurement, defined as

$$
A_{\text {meas }}(t)=A_{0} e^{-\left(\frac{t-t_{0}}{\tau}\right)}\left(1-e^{\frac{t_{a c q}}{\tau}}\right)
$$

Where $A_{0}$ is the initial certified activity of the source, $t-t_{0}$ is the source age (12 years 24 days, $9 \mathrm{~h}, 16 \mathrm{~min}$ ) (AEATECHNOLOGY set.365, 1 November 2005), $\tau$ is the half-life divided by $\ln (2)$, $\mathrm{t}_{\mathrm{acq}}$ is the acquisition time with dead-time included;
Table 4

Results obtained by ROOT-Hypermet comparison for ${ }^{133} \mathrm{Ba}$.

\begin{tabular}{lllll}
\hline & Peak n. & Energy $[\mathrm{keV}]$ & FWHM $[\mathrm{keV}]$ & Peak area \\
\hline \multirow{2}{*}{ ROOT } & 1 & $52.90 \pm 0.05$ & $1.02 \pm 0.05$ & $1015.8 \pm 27.4$ \\
& 2 & $79.06 \pm 0.01$ & $1.50 \pm 0.11$ & $2790.0 \pm 239.9$ \\
& 3 & $80.79 \pm 0.02$ & $0.94 \pm 0.05$ & $21125.4 \pm 676.0$ \\
& 4 & $160.44 \pm 0.25$ & $1.06 \pm 0.16$ & $122.3 \pm 27.5$ \\
& 5 & $223.12 \pm 0.55$ & $0.96 \pm 0.17$ & $58.9 \pm 15.1$ \\
& 6 & $276.08 \pm 0.04$ & $1.21 \pm 0.06$ & $2102.5 \pm 52.5$ \\
& 7 & $302.48 \pm 0.02$ & $1.15 \pm 0.08$ & $5224.5 \pm 94.0$ \\
Hypermet PC & 1 & $355.60 \pm 0.01$ & $1.29 \pm 0.03$ & $15343.0 \pm 199.4$ \\
& 2 & $383.40 \pm 0.05$ & $1.26 \pm 0.09$ & $2234.2 \pm 53.6$ \\
\hline & 2 & $79.74 \pm 0.05$ & $1.30 \pm 0.12$ & $2867.0 \pm 258.0$ \\
& 3 & $80.98 \pm 0.02$ & $0.90 \pm 0.03$ & $17247.0 \pm 448.4$ \\
& 4 & $160.62 \pm 0.13$ & $1.12 \pm 0.28$ & $352.0 \pm 112.3$ \\
& 5 & $223.03 \pm 0.13$ & $1.37 \pm 0.36$ & $180.0 \pm 56.7$ \\
& 6 & $276.40 \pm 0.03$ & $1.24 \pm 0.06$ & $2269.0 \pm 65.8$ \\
& 7 & $302.85 \pm 0.02$ & $1.24 \pm 0.04$ & $5261.0 \pm 99.9$ \\
& 8 & $356.01 \pm 0.01$ & $1.31 \pm 0.02$ & $15652.0 \pm 187.8$ \\
9 & $383.40 \pm 0.03$ & $1.35 \pm 0.04$ & $2134.0 \pm 49.1$ \\
\hline
\end{tabular}

2. The activity $A_{\epsilon} \epsilon(t)$ which is again evaluated during measurement [16], but is retrieved from the absolute efficiency through:

$$
A_{\varepsilon}(t)=\frac{C}{\tau} \frac{1}{P_{\gamma} \varepsilon_{a b s}} \frac{t_{a c q}}{t_{\text {live }}}
$$

Where $C$ is the number of counts under the photo-peak (peak area), $\mathrm{P}_{\gamma}$ is the gamma-emission probability, $\epsilon_{\mathrm{abs}}$ is the absolute efficiency consisting of the product of a (point-like) geometric factor and an intrinsic (polynomial) function, $t_{\text {live }}$ is the acquisition live-time;

3. The activity $A_{s t d}(t)$ at the beginning of the measurement, that is:

$$
A_{s t d}(t)=A_{0} e^{-\left(\frac{t-t_{0}}{\tau}\right)}
$$

For comparison, we completed the calculation using both Root and Hypermet PC. The results of this quantitative test are reported in Table 5.

As a remark of the validity of Eq. (1), and of its correct implementation in our ROOT routine, we fitted all the radionuclide peaks and calculated the residues for each of them. As we can see from Fig. 3, which reports a sample of such calculations, the residues present values mostly around zero, meaning that the function effectively fits the data.

\section{Conclusions}

The pulsed nature of the spallation source offers a chance to expand the PGAA technique into its T-PGAA version, which, in turn, discloses all limitations of closed-source, dedicated analysis packages, calling for an effort to redesign all analytical steps by means of a more flexible tool such as ROOT framework.

In this paper we presented a new software for $\gamma$ spectra data analysis developed using ROOT software, aiming to be preparatory for the analysis of the T-PGAA spectra performed at ISIS pulsed Neutron and Muon Source. In order to validate the new protocol for data analysis, we acquired $\gamma$ spectra from two calibrated radionuclides and performed the newly coded multi-peak analysis, comparing the new software results with those found using Hypermet PC. The new protocol gives reliable and comparable results to those from Hypermet. This work is a first, but fundamental, successful step towards the validation and application of this new software to T-PGAA spectra at spallation sources. Further applications of this new data analysis are ongoing on irradiated $\mathrm{Cu}$ based standards, whom spectra have been recently acquired through T-PGAA techniques at ISIS pulsed Neutron and Muon Source. 
Table 5

Root-Hypermet comparison of the activities calculated from: the absolute efficiency $\mathrm{A}_{\epsilon}$; the activity during the measurement $\mathrm{A}_{\text {meas }}$ and the standard activity $\mathrm{A}_{\text {std }}$.

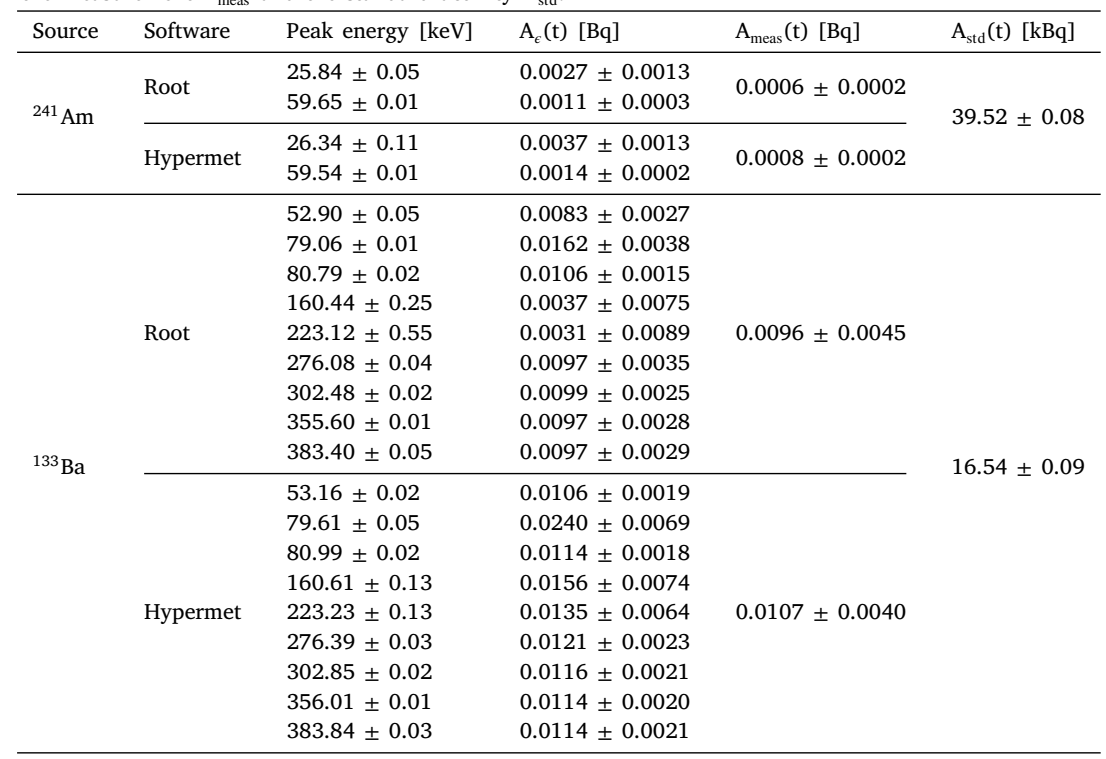
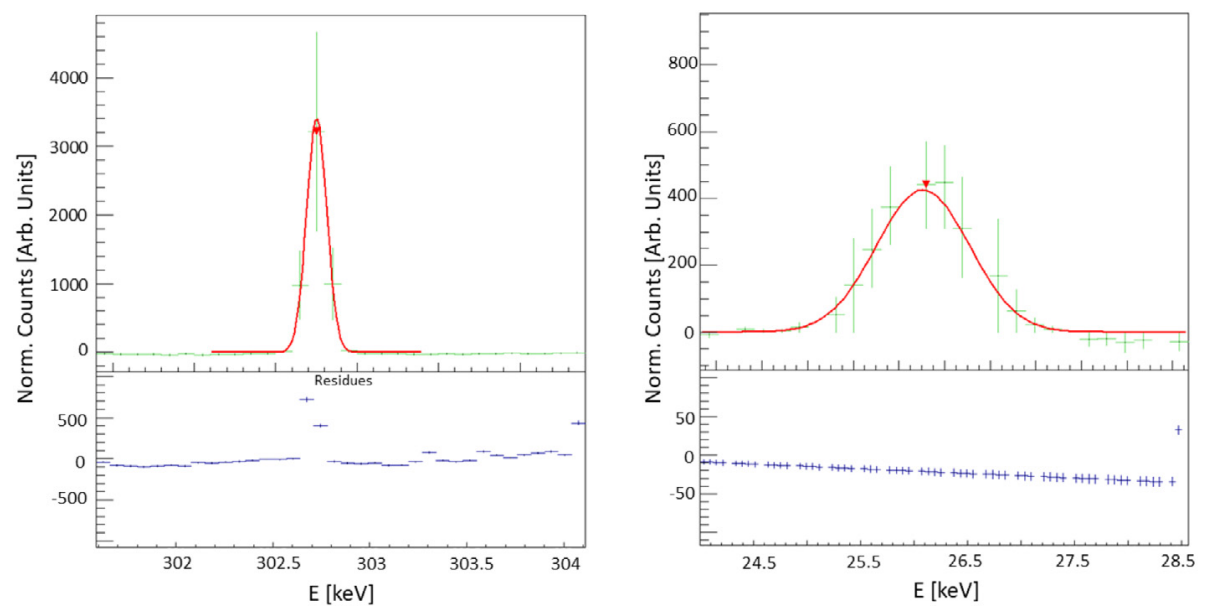

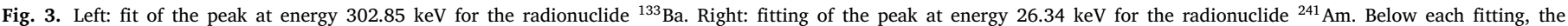
calculation of fit residues is reported.

\section{Acknowledgement}

This work was supported within the CNR-STFC agreement, Italy 2014-2020 (No 3420 2014-2020) concerning the collaboration in scientific research at ISIS pulsed Neutron and Muon Source.

\section{References}

[1] G. Festa, C. Andreani, L. Arcidiacono, G. Burca, W. Kockelmann, T. Minniti, R. Senesi, Characterization of gamma-ray background at IMAT beamline of ISIS spallation neutron source, J. Instrum. 12 (08) (2017) P08005.

[2] K.V. Tian, G. Festa, L. Szentmiklósi, B. Maróti, et al., Compositional studies of functional orthodontic archwires using prompt-gamma activation analysis at a pulsed neutron source, JAAS 32 (7) (2017) 1420-1427.

[3] G. Festa, L. Arcidiacono, A. Pappalardo, T. Minniti, C. Cazzaniga, A. Scherillo, C. Andreani, R. Senesi, Isotope identification capabilities using time resolved prompt gamma emission from epithermal neutrons, J. Instrum. 11 (2016) C03060.

[4] R. Henkelmann, Low energy gamma rays from thermal neutron capture, Z. Phys. 258 (1973) 315-320.

[5] R.W. Peelle, F.C. Maienschein, Spectrum of photons emitted in coincidence with fission of $235 u$ by thermal neutrons, Phys. Rev. C 3 (1) (1971) 373-390.

[6] G. Molnár, Handbook of Prompt Gamma Activation Analysis - with Neutron Beams, Springer, 2004.
[7] R. Brun, F. Rademakers, ROOT - An object oriented data analysis framework, in: Proceedings AIHENP'96 Workshop Lausanne Nucl Inst and Meth. in Physics a, Vol. 389, 1997.

[8] G.W. Phillips, K.W. Marlow, Program HYPERMET for automatic analysis of gamma-ray spectra from germanium detectors, Nucl. Instrum. Methods 72 (1976).

[9] Zs Revay, T. Belgya, P.P. Ember, G.L. Molnár, Recent developments in HYPERMET PC, J. Radoanalytical Nucl. Chem. 248 (2000).

[10] E. Fairstein, S. Wagner, IEEE Standard Test Procedures for Germanium GammaRay Detectors, IEEE std (New York, U.S.A.: The Institute of Electrical and Electronics Engineers, Inc.), 1996.

[11] A. Parmentier, L. Arcidiacono, R. Senesi, G. Romanelli, C. Andreani, J. Moir, G. Festa, Absolute efficiency calibration of a coaxial HPGe detector for quantitative PGAA and T-PGAA, J. Phys. Proc. Sect. eV Neutrons Matter S (2018).

[12] B. Fazekas, Zs. Rvay, J. Ostor, T. Belgya, G. Molnár, A. Simonits, A new method for determination of gamma-ray spectrometer non-linearity, NIM A 422 (1-3) (1999) 469-473.

[13] M. Morhác, J. Kliman, V. Matousek, M. Veselský, I. Turzo, Identification of peaks in multidimensional coincidence $\gamma$ ray spectra, NIM A 443 (2000) 108-125.

[14] M.A. Mariscotti, A method for identification of peaks in the presence of background and its application to spectrum analysis, NIM 50 (1967) 309-320.

[15] M. Morhác, J. Kliman, M. Jandel, L. Krupa, V. Matousek, Study of fitting algorithms applied to simultaneous analysis of large number of peaks in $\gamma$-ray spectra, Appl. Spectrosc. 57 (7) (2003) 753-760. 
[16] Z. Kis, B. Fazekas, J. Ostor, Z. Révay, T. Belgya, G.L. Molnár, L. Koltay, Comparison of efficiency functions for Ge gamma-ray detectors in a wide energy range, NIM A 418 (2-3) (1998) 374-386.

\section{Further reading}

[1] F. Pleasonton, R.L. Ferguson, H.W. Schmitt, Prompt gamma emitted in the thermal neutron induced fission of 235u, Phys. Rev. C 6 (3) (1972) 1023-1039. 